Supplement of Atmos. Meas. Tech. Discuss., 8, 2881-2912, 2015

http://www.atmos-meas-tech-discuss.net/8/2881/2015/

doi:10.5194/amtd-8-2881-2015-supplement

(C) Author(s) 2015. CC Attribution 3.0 License.

(c) (i)

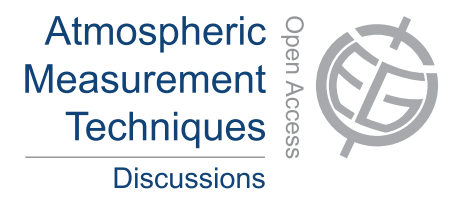

Supplement of

\title{
Plume-based analysis of vehicle fleet air pollutant emissions and the con- tribution from high emitters
}

\section{J. M. Wang et al.}

Correspondence to: J. M. Wang (jonm.wang@utoronto.ca) 


\section{Sampling and Instrument Details}

The gas analyzers Thermo Scientific models 42i, 48C, and 410i were calibrated before and after the measurement campaign using certified gas mixtures (Linde Canada Ltd., Mississauga, Ontario, Canada) for carbon monoxide (630 $\pm 13 \mathrm{ppm})$, carbon dioxide (99700 \pm $2000 \mathrm{ppm}$ ), and nitrogen oxide (79.6 $\pm 4 \mathrm{ppb}$ ); and a multi-gas calibrator (model 146i, Thermo Scientific, Waltham, MA). Negligible differences were observed between the pre- and postcampaign calibrations of the gas analyzers. The 48C CO analyzer was set to auto-zero every 12 hours to ensure quality assurance of the higher instrumental drift.

A six level calibration was run before and after the measurement period for the PTRTOF-MS using two standard VOC mixtures made by the National Air Pollution Surveillance Network at Environment Canada (Ottawa, Ontario) based on U.S. EPA-TO15 for 159 non-polar VOCs and 40 independently chosen polar VOCs. A 10 minute baseline zeroing audit period was performed every 12 hours to correct for the baseline. Data was exported using PTR Viewer 3.1.0.20 and converted from counts per second normalized to $\mathrm{H}_{3} \mathrm{O}+\mathrm{O}^{18}$ to mass concentration using the corresponding 6-level calibration curve. Furthermore, measurements were verified with coincident 24-hour integrated sampling in Summa stainless steel canisters analyzed by cryogenic preconcentration gas chromatography-mass spectrometry method as described in Wang et al. (2005). Concentrations were ensured to be below 20\% difference on average from each campaign between the PTR-TOF-MS and integrated sampling for BTEX.

The PASS-3 absorption measurement was converted to a black carbon mass concentration by linear correlation with thermal-optical analysis (Lab OC-EC, Sunset Laboratories, Tigard, OR) integrated over 2 hour periods that made coinciding measurements over the measurement campaign. The calculated conversion value from absorption $\left(\mathrm{Mm}^{-1}\right)$ to $\left(\mu \mathrm{g} / \mathrm{m}^{3}\right)$ was $0.31,0.27,0.26$, and 0.27 for fall, winter, spring, and summer campaigns respectively and expected to be specific for this study conditions.

Supplementary measurements included local temperature, relative humidity, and wind speed and direction were measured using a meteorological station (WXT520, Vaisala, Vantaa, Finland) at the local site. Vehicle counts was measured by a SmartSensor HD (SS-125, Wavetronix, Provo, UT) categorized as light-duty $(1.5-7 \mathrm{~m})$ and heavy-duty vehicles (7 - 18 $\mathrm{m}$ ) based on detected approximate length.

\section{Automatic Plume Identification Method}

Measurement data were processed and analyzed in Igor Pro 6.34. An algorithm was developed to automatically identify vehicle exhaust plumes based on the inflection points before and after a plume calculated from the slope of the $\mathrm{CO}_{2}$ signal averaged over 10 points. The start and end of the plume was automatically recorded for reference in the calculation of emission factors (Figure S1). Plumes were flagged as erroneous or uncaptured if it was shorter than $10 \mathrm{~s}$ or had a total integrated amount of $\mathrm{CO}_{2}$ over the plume period lower than $5 \mathrm{ppmv}\left(\sim 2 \mathrm{mg} \mathrm{C} \mathrm{m}^{-3}\right)$. Figure S1 presents plume cases ranging from more ideal (left panel) to less ideal (right panel), with various corresponding measured pollutant plumes. In some cases, there is no corresponding pollutant measurement due to negligible emission from the source. It is important to note that the 
CO trace has a lower time response in Figure S1 (middle panel), and in the automatic integration has a 20 second buffer on each side of the plume length.
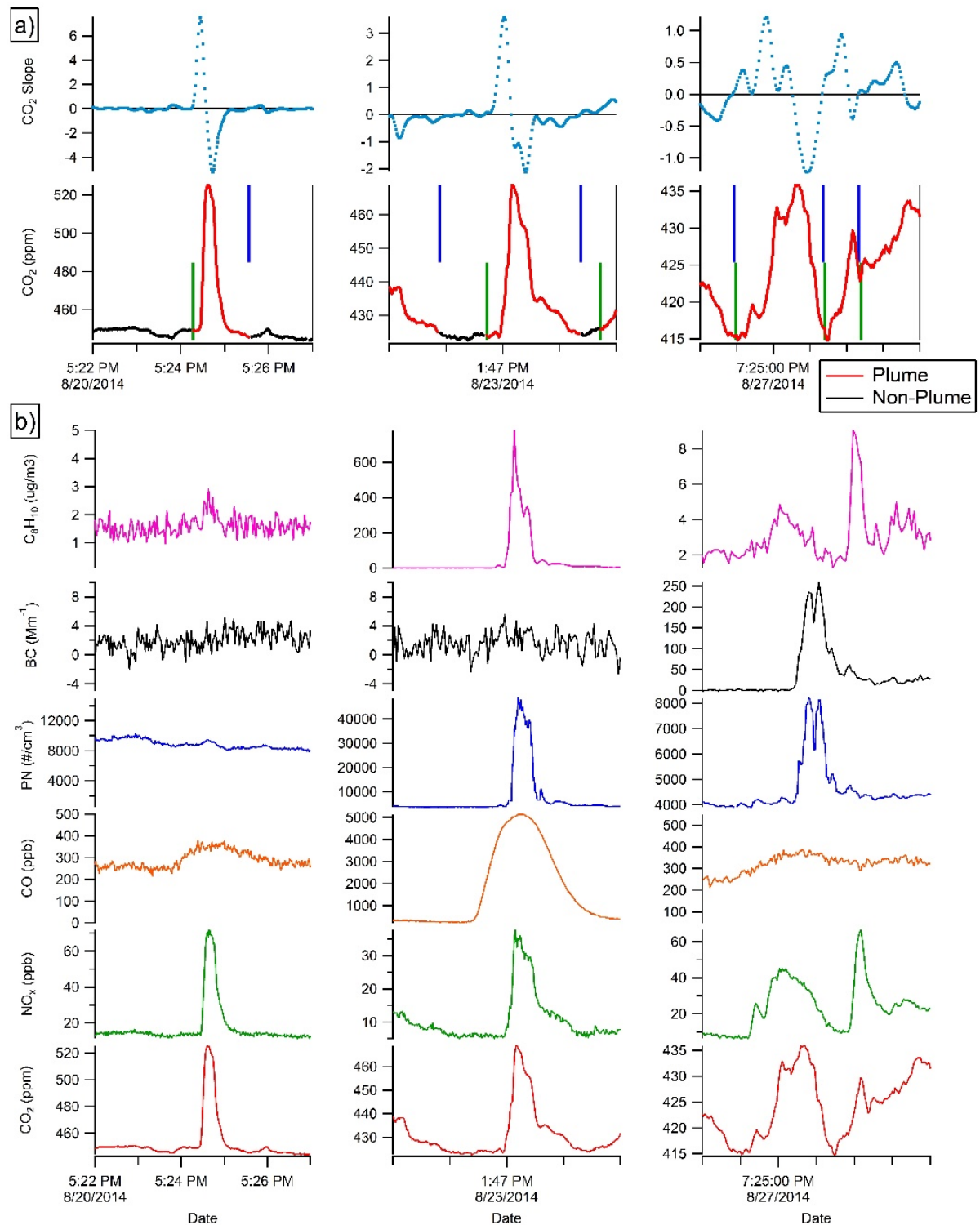

Figure S1: Time series of automatic plume identification results (a) of the $\mathrm{CO}_{2}$ trace separated as plume (red) and non-plume (black), with the sticks indicating beginning (green) and end (blue) of plume, and the corresponding slope of $\mathrm{CO}_{2}$. Measurement time series (b) with example plumes for different pollutants, illustrating pollutants which are or are not detected from the passing vehicle.

A minimum threshold based on the maximum and minimum signal calculated from multiple selected non-vehicle influenced ambient periods verified by video footage for each pollutant trace was used to ensure quality assurance of the calculated emission factors described in Section 2.3. Plumes that have a pollutant measurement below this threshold would have a below detection limit (BDL) emission factor for that specific pollutant (Figure S2). The detection of vehicle plumes were visually verified for selected periods over the measurement campaign for 
quality control of the automated identification. Both the automatic detection of the beginning and end of the plume, as well as the calculated pollutant EFs were within an acceptable range of manual calculation of EFs of the same plumes (see Individual EF Analysis).

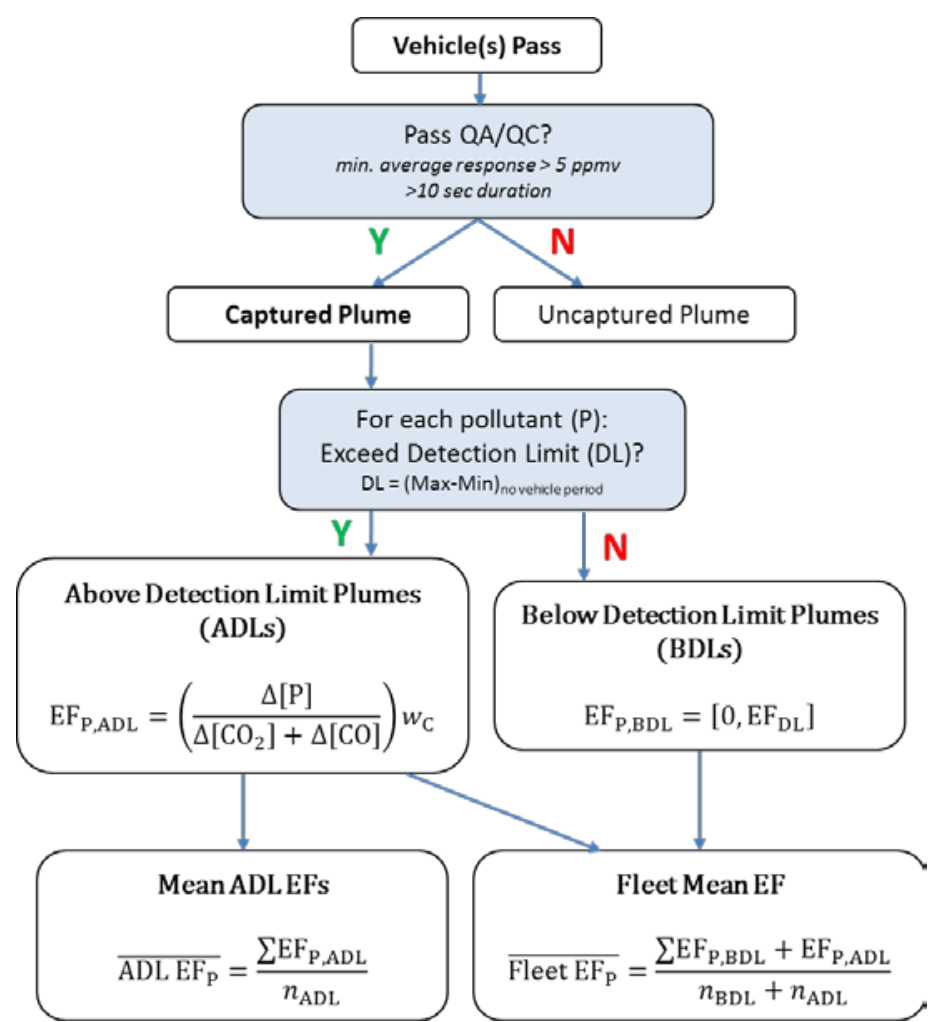

Figure S2: Process flow diagram of plume identification and capture quality assurance, criteria for ADL and BDL EFs, and the calculation for mean ADL and mean fleet EFs.

\section{Roadside versus Building Gradient Comparison}

The fuel-based method used calculate the emission factors have typically been conducted previously (Hansen and Rosen, 1990), and extensively in tunnel environments under strictly controlled conditions with the measurement inlet close to the tailpipe of vehicles (Ban-Weiss et al., 2009;Dallmann et al., 2012). One assumption made in the fuel-based measurement method is that very little dilution occurs from the tailpipe to the sample inlet, thus preserving the pollutants in the exhaust plume. However, this assumption had to be validated for this study given the increased travel distance and potential for higher dilution within the street canyon. This was tested for total PN from coincident measurements of $\mathrm{CO}_{2}$ using two identical LI-840s and CPCs (model 3788, TSI Inc., Shoreview, MN) at $3 \mathrm{~m}$ (roadside) from the main inlet and $15 \mathrm{~m}$ away from the roadway. At the end of each trial, the roadside instruments were moved to sample beside the main inlet to account for instrumental differences and any line losses. Particle number emission factors from 287 plumes were calculated independently from the two datasets (Figure S3), and percent difference was only 6\% based on the mean PN EFs. 


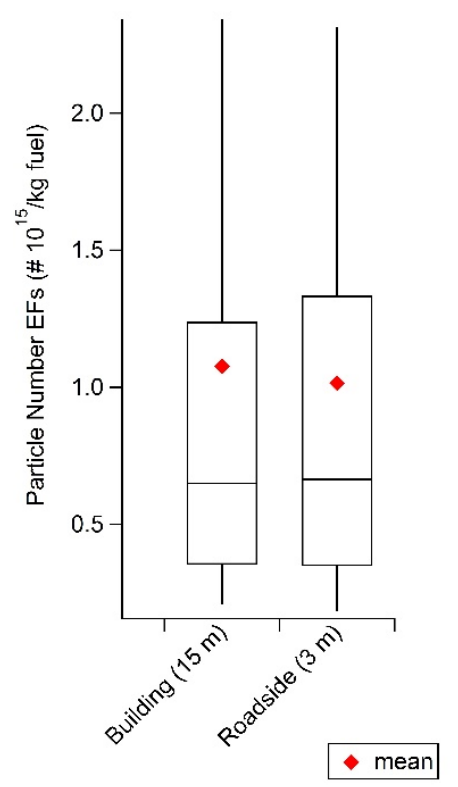

Figure S3: Particle number emission factors comparison between coincident roadside measurements and the main inlet $15 \mathrm{~m}$ from the roadway. Horizontal lines represent the median values, boxes represent the $75^{\text {th }}$ percentile and whiskers represent the $90^{\text {th }}$ percentile.

Assuming that the pollutants and $\mathrm{CO}_{2}$ are conserved within the plume and dilute similarly as they are transported from the tailpipe to the inlet, ideally there should be no correlation between the PN EFs and dilution ratio. A rough estimate of the dilution ratio from the gradient comparison calculated by assuming the output concentration of $\mathrm{CO}_{2}$ from the vehicles at $14 \%$ $(140,000 \mathrm{ppm} \mathrm{CO} 2)$ yielded no correlation between dilution ratio and PN EFs (Figure S4). This was assumed to be the case in many previous carbon balance fuel-based EF studies and has also been shown by Jayaratne et al. (2005). Assuming the same output of $\mathrm{CO}_{2}$, based on the average amount of $\mathrm{CO}_{2}$ measured in plumes, the mean dilution ratios from tailpipe to $3 \mathrm{~m}$ and $15 \mathrm{~m}$ were approximately 6000 and 10000 respectively. This indicates that although there may be roughly a $70 \%$ increase in dilution between $3 \mathrm{~m}$ and $15 \mathrm{~m}$, a large amount of dilution occurs on the road right after the tailpipe. Despite the large amount of dilution occurs, the PN EF values were observed to be consistent between $3 \mathrm{~m}$ and $15 \mathrm{~m}$ (Figure S3) within each trial, following the assumption that the exhaust plume is relatively well conserved within the street canyon. 


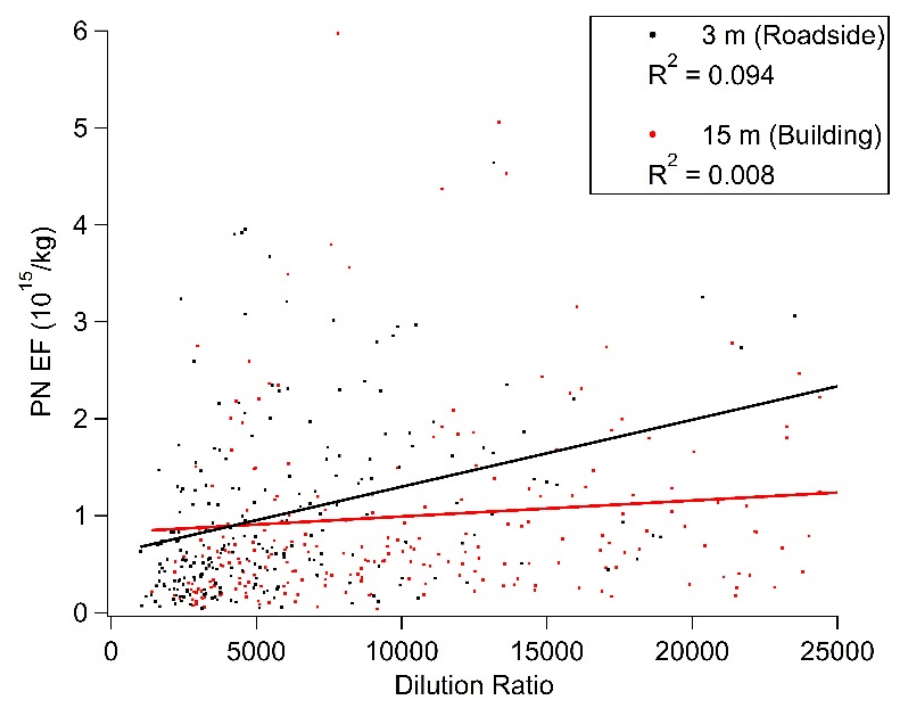

Figure S4: Scatter plot of calculated PN EFs from both $3 \mathrm{~m}$ (red) and $15 \mathrm{~m}$ (black) against dilution ratio determined by $\mathrm{CO}_{2}$.

\section{Repeated Drive-By Vehicle Tests and PN EF comparison}

In order to test the capture efficiency and accuracy of the measurements at the near-road site, a 2013 Ford Focus gasoline direct injection passenger vehicle was driven by the site testing four drive states; idle, cruise, acceleration, and braking. A total of 25 passes were made for each respective drive state, where a capture efficiency was calculated based on the number of passes where a plume was identified by $\mathrm{CO}_{2}$ and met quality assurance measures for a successful capture in contrast to an unsuccessful capture from no observable change in the pollutant signals. At $15 \mathrm{~m}$, the capture efficiencies were highest for idling in line with the inlet (52\%) compared to acceleration and braking (44\%), and cruise (42\%). Coincident measurements made at the roadside $(3 \mathrm{~m})$ had a higher average capture efficiency of $71 \%$. This indicates that there was a $25 \%$ decrease in capture efficiency at $15 \mathrm{~m}$, likely as a result of plumes missing the sample inlet or diluting pollutant concentrations below the sensitivity of the instrument.

The accuracy of the PN EFs were further tested by comparing measurements made in the real-world site to a 2012 Ford Focus engine tested with a dynamometer in laboratory conditions (Zimmerman et al., 2014). The average PN EFs from these laboratory tests agreed to within 14\% with the near road values determined in these drive-by tests, showing surprisingly good agreement given the two vastly different measurement conditions.

\section{Individual EF Analysis}

Individual plumes were selected from the measurement time series based on large magnitude pollutant plumes from the measurement period. These plumes did necessarily originated from high or heavy emitters as dilution can also play a critical role in the measured concentrations at the inlet. The selection basis used was visually obvious plumes in comparison with other plumes from the period based on high levels of any one or multiple pollutants. These short time interval periods were then verified as having no truck influence (cars) or having one or 
more trucks passing (cars and trucks) using video recordings of the passing fleet. Of the 152 heavy emitter plumes randomly chosen, 62 were cars and 88 were truck-influenced periods. Although the truck influenced-periods are more difficult to distinguish whether it is the truck or a poorly-tuned car that may be emitting higher levels of pollutants, the expected lag time was typically matched to the passing truck.

A subset of 75 plumes were selected randomly from the individual plume analysis and EFs were calculated using two techniques. The automated technique has been described in Section 2.3. The manual technique identified plumes on a visual basis from the time series data. The plume was then background subtracted by selecting a minimum point from the plume period, manually integrated and an EF was calculated in a similar manner as the automated technique. On average, the mean percent difference between manual and automatic methods was 8\% and within acceptable limits given the differences in the two methods (Table S1).

Major deviations of the EF values (17\% for BC) was most influenced by noisier measurement signals, which resulted in the baseline determination for background subtraction. In the automated method, noisy signals are dealt with by taking an average of the lower value points in order to determine a minimum value. Additionally, effective sensitivities were determined for the instrument in order to eliminate the effect of noise on lower EF values. Testing this on some of the manually calculated BC EFs resulted in a decrease in mean percent different by a factor of two. Other deviations between the two methods were the result of the plume identification differences, where although both methods were consistent in start times, the length of the plume was highly subjective in the manual method.

Table S1: Mean percent difference of calculated EFs using manual and automatic techniques.

\begin{tabular}{|l|l|}
\hline Pollutant & $\begin{array}{l}\text { Mean Percent } \\
\text { Difference (\%) }\end{array}$ \\
\hline & \\
\hline $\mathrm{NO}_{\mathrm{x}}$ & $6.9 \%$ \\
\hline $\mathrm{CO}$ & $8.0 \%$ \\
\hline $\mathrm{PN}$ & $7.1 \%$ \\
\hline $\mathrm{BC}$ & $16.9 \%$ \\
\hline $\mathrm{CH}_{3} \mathrm{OH}$ & $9.7 \%$ \\
\hline $\mathrm{C}_{6} \mathrm{H}_{6}$ & $8.6 \%$ \\
\hline $\mathrm{C}_{7} \mathrm{H}_{8}$ & $7.2 \%$ \\
\hline $\mathrm{C}_{8} \mathrm{H}_{10}$ & $6.5 \%$ \\
\hline
\end{tabular}

\section{Pollutant EF Distributions}

The mean ADL EFs described in Figure S2 are dependent on the sensitivity of the measurement methods used for each respective pollutant. These ADL EF values are not representative of the entire fleet, but rather the subsection of the fleet with detectable emissions of the specific pollutant. This becomes especially important in a plume-by-plume analysis, where selectively identifying plumes based on measurably higher pollutant levels may preferentially target heavier emitting vehicles. Time-integrated sampling is only limited by the detection limits 
of the measurement technique because all emissions are inherently captured by each sample. However, proper inclusion of BDL values is crucial in plume-by-plume analysis, where in order to represent the entire detectable fleet, a representative sample that includes BDL plumes needs to be taken into consideration, as done in this study. A considerable difference is observed when comparing fleet mean EFs with mean EFs calculated only using ADL EFs (Table S2). In particular, pollutants with a high percentage of BDL EFs including BC, CO, and BTEX EFs are nearly half of their respective mean ADL EF values.

Table S2: Fleet-average emission factors from detectable plumes

\begin{tabular}{|l|l|l|l|l|l|}
\hline Pollutant & $\begin{array}{l}\text { Fleet Mean EFs } \\
\pm 95 \% \text { CI }\end{array}$ & $\begin{array}{l}\text { Mean ADL EFs } \\
\pm 95 \% \text { CI }\end{array}$ & $\begin{array}{l}\text { Geometric Mean } \\
\text { ADL EFs }\end{array}$ & EF Unit & BDL EFs $^{\mathrm{c}}$ \\
\hline $\mathrm{NO}_{\mathrm{x}}$ & $2.27-2.32 \pm 0.02$ & $3.16 \pm 0.04$ & 2.40 & $\mathrm{~g} \mathrm{~kg}^{-1}$ & $28 \%$ \\
\hline $\mathrm{CO}$ & $13.6-14.2 \pm 0.5$ & $41.3 \pm 1.6$ & 30.2 & $\mathrm{~g} \mathrm{~kg}^{-1}$ & $67 \%$ \\
\hline $\mathrm{PN}$ & $0.77-0.77^{\mathrm{d}} \pm 0.01$ & $1.03 \pm 0.03$ & 0.55 & $\# 0^{15} \mathrm{~kg}^{-1}$ & $26 \%$ \\
\hline $\mathrm{BC}$ & $0.048-0.061 \pm 0.001$ & $0.133 \pm 0.005$ & 0.072 & $\mathrm{~g} \mathrm{~kg}^{\mathrm{e}}$ & $65 \%$ \\
\hline $\mathrm{CH}_{3} \mathrm{OH}$ & $1.65-1.67 \pm 0.09$ & $3.50 \pm 0.23$ & 1.42 & $\mathrm{~g} \mathrm{~kg}^{-1}$ & $52 \%$ \\
\hline $\mathrm{C}_{6} \mathrm{H}_{6}$ & $0.052-0.054 \pm 0.0005$ & $0.098 \pm 0.001$ & 0.080 & $\mathrm{~g} \mathrm{~kg}^{-1}$ & $47 \%$ \\
\hline $\mathrm{C}_{7} \mathrm{H}_{8}$ & $0.159-0.162 \pm 0.006$ & $0.297 \pm 0.023$ & 0.182 & $\mathrm{~g} \mathrm{~kg}^{-1}$ & $46 \%$ \\
\hline $\mathrm{C}_{8} \mathrm{H}_{10}$ & $0.151-0.155 \pm 0.002$ & $0.286 \pm 0.004$ & 0.213 & $\mathrm{~g} \mathrm{~kg}^{-1}$ & $47 \%$ \\
\hline
\end{tabular}

${ }^{a}$ Mean EFs for all identified plumes including BDL EFs as zero and calculated with the operational detection limit, ${ }^{b}$ mean EFs with all BDL EFs removed respective to each pollutant, ${ }^{c}$ percentage of EFs treated as BDL and given a zero value, ${ }^{d}$ range for fleet mean PN EFs is negligible due to the low operational detection limit of the CPC, ${ }^{e}$ BC EFs from the PASS-3 only had $75 \%$ data coverage due to automatic zeroing of the instrument.

Pollutant EFs were found to be log normally distributed, with the pollutants having one mode (Figure S5). It is important to note that the BDL or zero/detection limit value EFs are excluded from these distributions but are included as percentages in Table S1. Some of the pollutant EFs were limited less by the measurement limit of detection, as there is an apparent decrease in the distribution at lower EF levels. However, in the distribution of certain pollutant EFs such as BC, CO, and VOCs a truncated distribution is apparent, most likely associated with poorer sensitivities. This is most apparent in the EF distribution of $\mathrm{BC}$, where the mode is close to zero, thus making it difficult to determine whether this is the true mode or if it is below the detection limit of the PASS-3. 

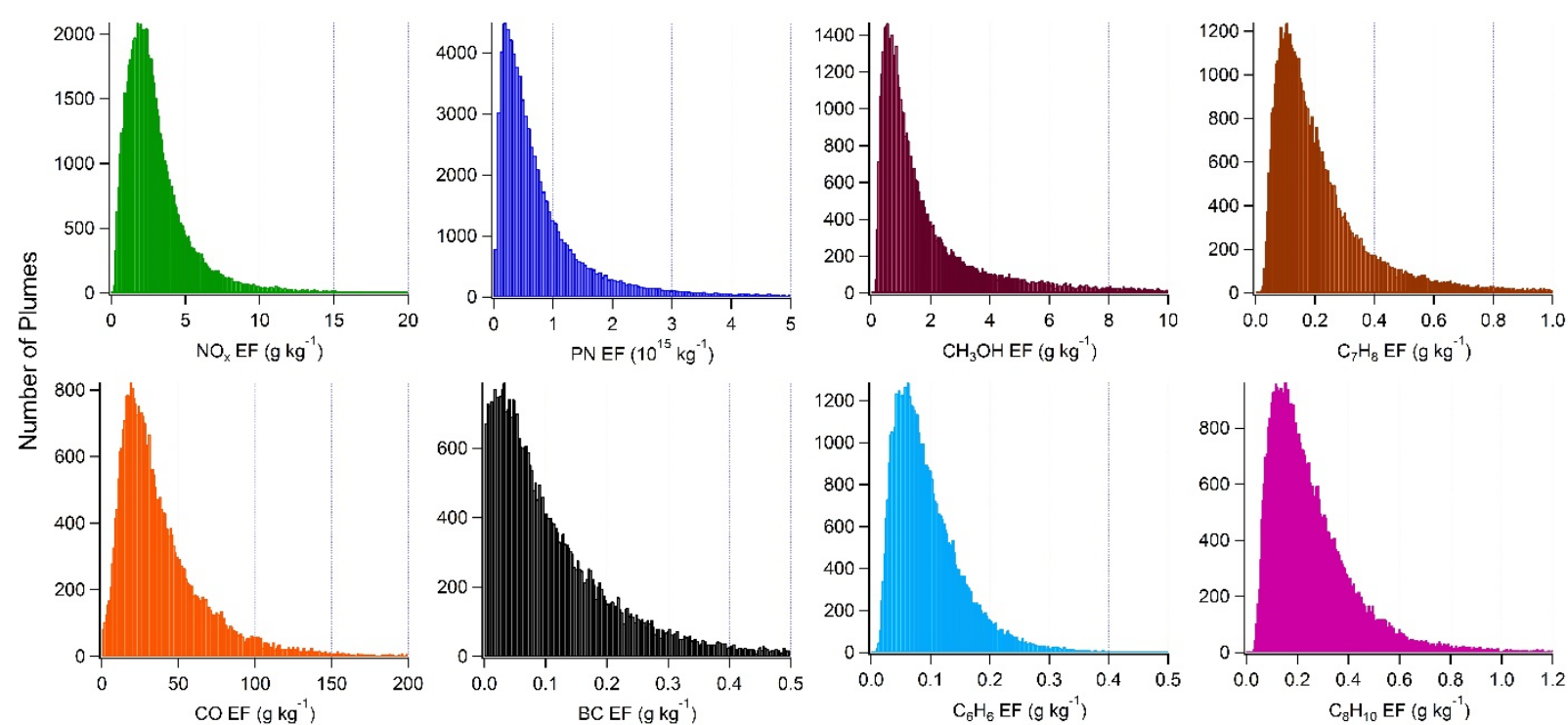

Figure S5: Emission Factor distributions for pollutants nitrogen oxides, black carbon, particle number, carbon monoxide, methanol, benzene, toluene, and ethylbenzene+xylenes.

The stacked plot (Figure S6), similar to the Figure 2 in the text, used the operational detection limit rather than zero values to represent the BDLs in order to provide a more conservative value for the percent contribution of emissions from higher EF emitters. Although the distributions of the contributions remain similar to those shown in Figure 4 of the text, one noticeable change is in the distribution for BC, which is much lower. This is the result of a relatively higher defined detection limit compared to the other pollutant measurements as a result of noise from the PASS-3, which shifts the distribution towards the BDL EFs for BC. It is unexpected that a large proportion of the fleet would emit significant amounts of $\mathrm{BC}$, thus these BDL EFs should be closer to zero. In addition to the higher BDL EF values as result of using the detection limit for calculation, the sheer number of BDL EFs of BC further inflates the lower portion of the distribution. Thus, this figure was included in the supporting information as reference and not included in the text. 


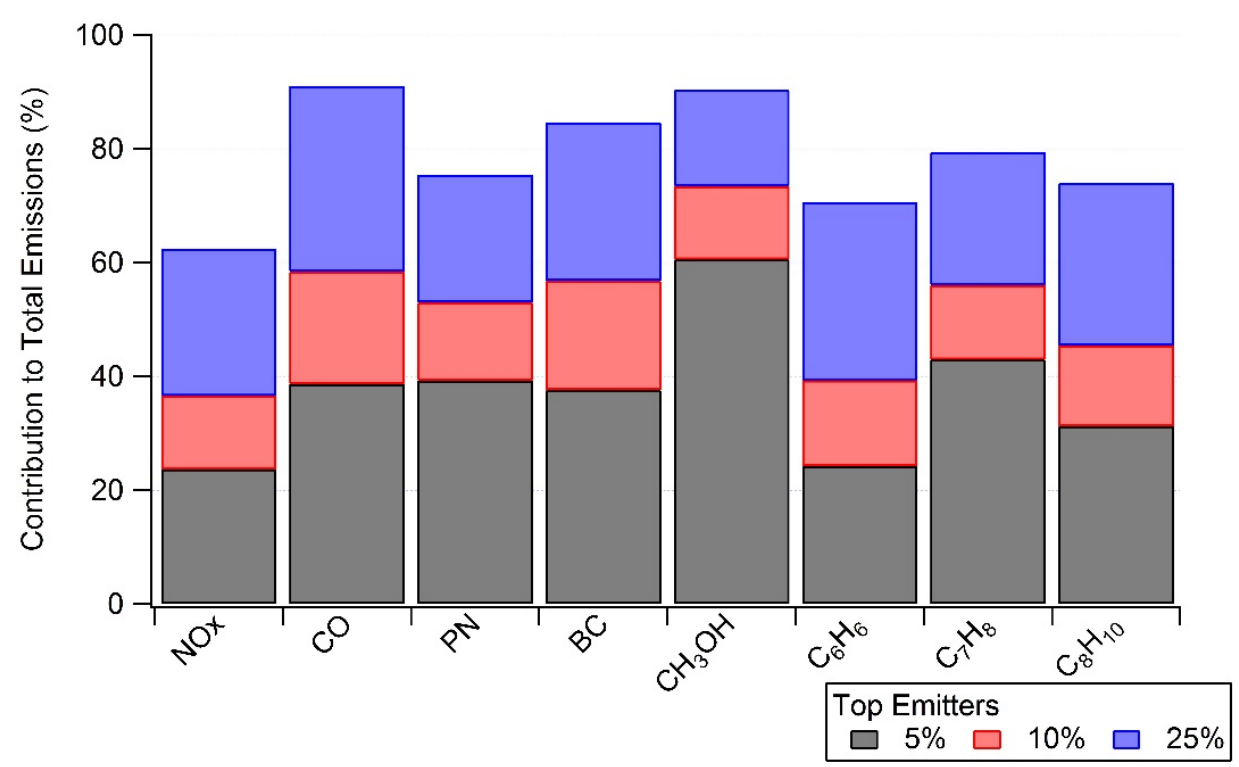

Figure S6: Stacked bar plot showing the contribution of heavy emitters in percentage based on the top 5,10 , and $25 \%$ of detectable plumes used to calculate EFs and operational detection limit to calculate BDLs.

\section{Ontario’s Emissions Testing Program}

Vehicle emissions in southern Ontario are regulated by the provincial government through "Drive Clean”, an emissions testing program for in-use vehicles similar to the U.S. EPA. For LDVs, emissions testing of in-use vehicles is equivalent of U.S. EPA Tier 1 standards, and are conducted for vehicles older than 7 years. Certain exemptions apply including vehicles manufactured before 1988, hybrid vehicles and motorcycles, and geographical locations outside of the Windsor-Ottawa corridor. Gasoline vehicles undergo mandatory testing on a dynamometer for $\mathrm{CO}, \mathrm{NO}_{\mathrm{x}}$, and hydrocarbon emissions every two years. Additionally, light-duty diesel vehicles are also tested using a visible smoke test for exhaust opacity, a measure for particulate matter in the vehicle exhaust. For HDVs, the program is modelled after U.S. EPA 2004 and 2007 HDV emissions standards based on model year, however only an exhaust opacity test is conducted for PM emissions. Since January 2013, the Drive Clean program began solely using the onboard diagnostic for post-1997 LDVs to infer emissions quality (McCarter, 2012;MoE, 2010).

\section{References}

Ban-Weiss, G. A., Lunden, M. M., Kirchstetter, T. W., and Harley, R. A.: Measurement of Black Carbon and Particle Number Emission Factors from Individual Heavy-Duty Trucks, Environ. Sci. Technol., 43, 1419-1424, doi:10.1021/es8021039, 2009.

Dallmann, T. R., DeMartini, S. J., Kirchstetter, T. W., Herndon, S. C., Onasch, T. B., Wood, E. C., and Harley, R. A.: On-Road Measurement of Gas and Particle Phase Pollutant Emission Factors for Individual Heavy-Duty Diesel Trucks, Environ. Sci. Technol., 46, 8511-8518, doi:10.1021/es301936c, 2012.

Hansen, A. D. A., and Rosen, H.: Individual Measurements of the Emission Factor of Aerosol Black Carbon in Automobile Plumes, J. Air. Waste. Manage., 40, 1654-1657, doi:10.1080/10473289.1990.10466812, 1990. 
Jayaratne, E. R., Morawska, L., Ristovski, Z. D., and Johnson, G. R.: The use of carbon dioxide as a tracer in the determination of particle number emissions from heavy-duty diesel vehicles, Atmos. Environ., 39, 6812-6821, doi:10.1016/j.atmosenv.2005.07.060, 2005.

McCarter, J.: 2012 Annual Report, Office of the Auditor General of Ontario, 107-128, 2012.

MoE: Drive Clean Guide - Emissions Standards, Emission Test Methods, and Technical Information Relating to Ontario Regulation 361/98 As Amended, Ontario Ministry of the Environment, Toronto, Ontario, 1-20, 2010.

Wang, D., Fuentes, J. D., Travers, D., Dann, T., and Connolly, T.: Non-methane hydrocarbons and carbonyls in the Lower Fraser Valley during PACIFIC 2001, Atmos. Environ., 39, 5261-5272, doi:10.1016/j.atmosenv.2005.05.035, 2005.

Zimmerman, N., Jeong, C. H., Wang, J. M., Ramos, M., Wallace, J. S., and Evans, G. J.: A sourceindependent empirical correction procedure for the fast mobility and engine exhaust particle sizers, Atmos. Environ., doi:10.1016/j.atmosenv.2014.10.054, 2014. 